\title{
Bounds on Capital Requirements For Bivariate Risk with Given Marginals and Partial Information on the Dependence
}

\begin{abstract}
Nelsen et al. [20] find bounds for bivariate distribution functions when there are constraints on the values of its quartiles. Tankov [25] generalizes this work by giving explicit expressions for the best upper and lower bounds for a bivariate copula when its values on a compact subset of $[0,1]^{2}$ are known. He shows that they are quasi-copulas and not necessarily copulas. Tankov [25] and Bernard et al. [3] both give sufficient conditions for these bounds to be copulas. In this note we give weaker sufficient conditions to ensure that both bounds are simultaneously copulas. Furthermore, we develop a novel application to quantitative risk management by computing bounds on a bivariate risk measure. This can be useful in optimal portfolio selection, in reinsurance, in pricing bivariate derivatives or in determining capital requirements when only partial information on dependence is available.

Keywords

Copulas • Fréchet-Hoeffding bounds - Capital requirements

MSC: 62E99,62H99, 62P05

(C) 2013 Carole Bernard et al., licensee Versita Sp. z o. o. This work is licensed under the Creative Commons Attribution-NonCommercial NoDerivs license, which means that the text may be used for non-commercial purposes, provided credit is given to the author.
\end{abstract}

\author{
Carole Bernard ${ }^{1 *}$, Yuntao $\mathrm{Liu}^{2 \dagger}$, Niall MacGillivray ${ }^{3 \neq}$ \\ Jinyuan Zhang $4 \S$ \\ 1 Department of Statistics and Actuarial Science \\ at the University of Waterloo \\ 2 University of California \\ 3 University of Waterloo \\ 4 University of British Columbia
}

Received 5 May 2013

Accepted 8 October 2013

The goal of this paper is to generalize the Fréchet-Hoeffding bounds when additional information on the dependence is available. Earlier work on this topic dates back to Rachev and Rüschendorf [21] who derive bounds on copulas when there are inequality constraints. Nelsen et al. [19] find best possible bounds on the bivariate distribution function of continuous variables with given marginals and given measure of association such as Kendall's tau or Spearman's rho. Nelsen [18] derives best possible bounds when the copula is known at a specific point. Our objective in this paper is to extend this literature to the case when the copula is known in more than one point and to show how these bounds can be useful in quantifying dependence misspecification. Assuming that marginals are given and that the dependence is unspecified, or partly unspecified, bounds on copulas can be indeed used to quantify this type of model risk.

To do so, we make use of the recent work of Tankov [25] who generalize Nelsen [18]'s result by giving explicit expressions for the best upper and lower bounds for a bivariate copula when its values on a compact subset of $[0,1]^{2}$ are known (see also Sadooghi-Alvandi et al. [23]). Tankov shows that they are quasi-copulas and not necessarily copulas. In this paper, we focus on deriving bounds on copulas that are also copulas. The first section focuses on finding simple conditions to ensure that Tankov's bounds are copulas. When the bounds are not copulas, it is possible to approximate them by a copula. The second section illustrates a method to find the best copula for the uniform norm that approximates the

\footnotetext{
* E-mail: c3bernar@uwaterloo.ca (Corresponding author), Tel: 00151988845678 ext: 35505.

+ E-mail: y253liu@berkeley.edu

* E-mail: niallmacgillivray@gmail.com

§E-mail: 0527zhangjinyuan@gmail.com
} 
bounds. For example, these bounds can be used to find the worst dependence and the best dependence to maximize or minimize a risk measure, which respects concordance order. This is directly useful to quantify model risk as we illustrate in the paper by finding bounds on capital requirements or on insurance premiums when the dependence is partially known.

We work on bounds for two-dimensional copulas. One reason is that bounds on two-dimensional copulas can easily be transfered on bounds on risk measures (as long as they are consistent with concordance order). In higher dimensions, it is not clear how this property can be extended. In particular the recent work of Embrechts, Puccetti and Rüschendorf [10] and Bernard, Jiang and Wang [4] show that the dependence structure of the minimum and maximum convex order bounds are "marginal" dependent ${ }^{1}$ and thus cannot be obtained from bounds on copulas only. Although the problem of model risk and dependence misspecification is also relevant in higher dimensions, alternative methods are needed and it is outside the scope of this paper. In addition, obtaining expressions for bounds on copulas in a multidimensional setting is challenging without any constraints on the dependence as there are almost no results on the lower Fréchet-Hoeffding bound for multidimensional copulas with $n \geqslant 3$. Our focus in this paper is on two-dimensional copulas. Bounds on copulas in two dimensions are extremely useful to solve portfolio selection problems, as it appears clearly in Bernard et al. $[1,2]$ where the key element is the dependence between a portfolio and a market index. When there are no constraints, it is shown that optimal portfolios (respectively worst portfolios) are generally comonotonic with the market portfolio (respectively anti-monotonic). However when investors have state-dependent preferences and look for optimal portfolios that verify additional constraints then the problem of solving the optimal portfolio amounts to solving an upper and lower bound on copulas with constraints. The optimal portfolio can be constructed if and only if the bound on copulas is a copula (and not only a quasi copula) and corresponds thus to a feasible dependence structure.

Recall that $Q:[0,1]^{2} \rightarrow[0,1]$ is a quasi-copula if it satisfies the following three properties. (1) For all $u \in$ $[0,1], Q(0, u)=Q(u, 0)=0$, and $Q(1, u)=Q(u, 1)=u$ (boundary conditions). (2) $Q$ is non-decreasing in each argument. (3) For all $u_{1}, v_{1}, u_{2}, v_{2} \in[0,1],\left|Q\left(u_{2}, v_{2}\right)-Q\left(u_{1}, v_{1}\right)\right| \leqslant\left|u_{2}-u_{1}\right|+\left|v_{2}-v_{1}\right|$ (Lipschitz property). If, in addition, $Q$ is 2 -increasing ${ }^{2}$ then it is a copula. More details on quasi-copulas can be found for example in Genest et al. [12]. Let $\mathcal{S}$ denote a compact subset of the unit square $[0,1]^{2}$. Tankov [25] shows that $A^{\mathcal{S}, Q}$ and $B^{\mathcal{S}, Q}$ defined by

$$
\begin{aligned}
& A^{\mathcal{S}, Q}(u, v)=\min \left\{u, v, \min _{(a, b) \in \mathcal{S}}\left\{Q(a, b)+(u-a)^{+}+(v-b)^{+}\right\}\right\}, \\
& B^{\mathcal{S}, Q}(u, v)=\max \left\{0, u+v-1, \max _{(a, b) \in \mathcal{S}}\left\{Q(a, b)-(a-u)^{+}-(b-v)^{+}\right\}\right\},
\end{aligned}
$$

where $(u, v) \in[0,1]^{2}$, are the best possible upper (resp. lower) bounds for the set of all quasi-copulas $Q^{\prime}$ such that $Q^{\prime}(a, b)=Q(a, b)$ for all $(a, b) \in \mathcal{S}$ (see Tankov [25], Theorem 1). When $\mathcal{S}$ is the empty set, $B^{\mathcal{S}, Q}(u, v):=\max (0, u+v-1)$ and $A^{\mathcal{S}, Q}(u, v):=\min (u, v)$ are the Fréchet-Hoeffding bounds.

Tankov [25] proves that a sufficient condition for $A^{\mathcal{S}, Q}$ (resp. $B^{\mathcal{S}, Q}$ ) to be a copula is to suppose that $\mathcal{S}$ is non-increasing (resp. non-decreasing). Bernard et al. [3] extend this result and show that when $Q$ is a copula, $A^{\mathcal{S}, Q}$ (resp. $B^{\mathcal{S}, Q}$ ) is a copula when $\mathcal{S}$ is a compact set satisfying some additional conditions, precisely some "monotonicity" and "connectivity" conditions ${ }^{3}$. For instance, when $\mathcal{S}$ is a rectangle then both $A^{\mathcal{S}, Q}$ and $B^{\mathcal{S}, Q}$ are copulas.

Our first contribution is to derive weaker and simpler conditions than Tankov [25] and Bernard, Jiang and Vanduffel [3] to ensure that $A^{\mathcal{S}, Q}$ and $B^{\mathcal{S}, Q}$ are both copulas. This is particularly important as it is then possible to construct explicitly the dependence that maximizes, respectively minimizes a given bivariate risk measure. As discussed above, it allows for

1 When changing the marginal distribution, the worst possible dependence may be obtained for different copulas.

${ }^{2} Q$ is 2-increasing if $V_{Q}(R)=Q\left(u_{2}, v_{2}\right)+Q\left(u_{1}, v_{1}\right)-Q\left(u_{1}, v_{2}\right)-Q\left(u_{2}, v_{1}\right) \geqslant 0$ for every rectangle $R=\left[u_{1}, u_{2}\right] \times\left[v_{1}, v_{2}\right] \subseteq$ $[0,1]^{2}$.

3 The precise result is given as follows. Denote by $\mathcal{S}_{1}$ the set obtained by the first variable projection of the compact set $\mathcal{S}$ and $\mathcal{S}_{2}$ as the second variable projection. Define the two following functions $\gamma_{1}$ from $\mathcal{S}_{1}$ to $\mathcal{S}_{2}$ as $\gamma_{1}(u)=\min \{v \mid(u, v) \in \mathcal{S}\}$ and $\gamma_{2}$ from $\mathcal{S}_{1}$ to $\mathcal{S}_{2}$ as $\gamma_{2}(u)=\max \{v \mid(u, v) \in \mathcal{S}\}$. For a copula $Q$ and a compact set $\mathcal{S} \subseteq[0,1]^{2}$ with both $\gamma_{1}$ and $\gamma_{2}$ non-decreasing functions, and satisfying the following property $\forall\left(u, v_{0}\right),\left(u, v_{1}\right) \in \mathcal{S},\left(u, \frac{v_{0}+v_{1}}{2}\right) \in \mathcal{S}$, then $B^{\mathcal{S}, Q}$ is a copula. A similar result is available for $A^{\mathcal{S}, Q}$. 
instance to extend earlier works on optimal portfolio selection by Bernard, Boyle and Vanduffel [1] and Bernard and Vanduffel [5].

Our second contribution is to derive a novel application to quantitative risk management and financial engineering. The idea builds on the observation that the dependence between two companies and two variables is often state-dependent. There is empirical evidence that regimes exist in the financial market and that the dependence structure depends on the regime. For example, Stoeber and Czado [24] find that regime switches are present in the dependence structure of various data sets and show that regime switching models can describe accurately inhomogeneity during times of crisis. Observe also that many sectors and companies appear to be independent unless systemic events happen (causing common shocks in the market). In practice, the dependence may not be known in all regimes. For example the dependence in the financial market can easily be observed in normal conditions but the lack of data makes it difficult to estimate it during extreme conditions. Our results can be applied to compute bounds on a bivariate risk measure when such partial information on the dependence is known. This turns out to be useful in pricing reinsurance claims in a multivariate setting, in pricing bivariate derivatives or in determining capital requirements. We are also able to exhibit explicitly the "worst" (resp. the "best") copula which makes the underlying risk the largest (resp. the smallest).

Our last contribution is to study what happens when at least one of the two bounds is not a copula. We illustrate our approach when there are exactly two constraints on the copula. Nelsen [18] derives best possible bounds when there is one constraint but the problem is more difficult when there is more than one constraint as either the lower bound or the upper bound may not be a copula. We derive explicit expressions for the smallest copula above the lower bound and the largest copula below the upper bound for the infinite norm when there are exactly two constraints. This can be useful to approximate the lower or upper bound when they are not sharp (that is when they could not be attained by copulas). Although the work is done with two constraints a similar technique can be applied when there are more than two constraints on the copula.

Section 1 gives a sufficient simple condition to ensure that both $A^{\mathcal{S}, Q}$ and $B^{\mathcal{S}, Q}$ are copulas. We propose an application to computing bounds on capital requirements and illustrate our findings with some numerical examples in Section 2. Section 3 focuses on the case when there are exactly two constraints on the copula and gives explicit expressions for the smallest copula above the lower bound and the largest copula below the upper bound for the infinite norm.

\section{Sufficient Conditions for Both Lower and Upper Bounds to be Copulas}

This section is an extension of Theorem 1 of Tankov [25] and Sadooghi-Alvandi et al. [23]. To prove our results, we use the following well-known lemma. Its proof is omitted as it is proved in Lemma 3.1 of Durante and Jarworski [8] or Lemma 1 of Bernard, Jiang and Vanduffel [3].

\section{Lemma 1.}

Assume $f:[0,1]^{2} \mapsto \mathbb{R}$ is 2-increasing, non-decreasing in each argument and satisfies the Lipschitz property. Define the function $g:[0,1]^{2} \mapsto \mathbb{R}$ as $g(u, v)=\max \{f(u, v), W(u, v)\}$, where $W(u, v)=\max \{u+v-1,0\}$ is the anti-monotonic copula. Then $g$ is also 2-increasing, non-decreasing in each argument and satisfies the Lipschitz property.

\section{Theorem 2.}

If $\mathcal{S}$ is a compact set satisfying the following property:

$$
\forall\left(a_{0}, b_{0}\right) \in \mathcal{S}, \forall\left(a_{1}, b_{1}\right) \in \mathcal{S}, \quad\left(a_{0}, b_{1}\right) \in \mathcal{S},\left(a_{1}, b_{0}\right) \in \mathcal{S}
$$

Furthermore, suppose $Q$ is a quasi-copula such that $\forall\left(a_{0}, b_{0}\right),\left(a_{1}, b_{1}\right) \in \mathcal{S}$ with $a_{0}<a_{1}, b_{0}<b_{1}$, we have

$$
Q\left(a_{1}, b_{1}\right)+Q\left(a_{0}, b_{0}\right)-Q\left(a_{0}, b_{1}\right)-Q\left(a_{1}, b_{0}\right) \geqslant 0
$$

then $A^{\mathcal{S}, Q}$ and $B^{\mathcal{S}, Q}$ are copulas. Note that condition (3) is automatically satisfied when $Q$ is a copula.

Proof. The proof of Theorem 2 is inspired from Bernard, Jiang and Vanduffel [3]. It is sufficient to prove that $B^{\mathcal{S}, Q}$ is a copula as the proof for $A^{\mathcal{S}, Q}$ follows immediately. Since Tankov [25] already proved that $B^{\mathcal{S}, Q}$ is a quasi-copula, all 
that remains is to establish that it is also 2-increasing. Define $f^{\mathcal{S}, Q}$ as

$$
f^{\mathcal{S}, Q}(u, v)=\max _{(a, b) \in \mathcal{S}}\left\{Q(a, b)-(a-u)^{+}-(b-v)^{+}\right\}
$$

Consider any rectangular area $R=\left[u_{1}, u_{2}\right] \times\left[v_{1}, v_{2}\right] \subset[0,1]^{2}$. To establish the 2-increasing property of $B^{\mathcal{S}, Q}$, it first must be shown that $f^{\mathcal{S}, Q}$ is 2 -increasing, that is $V_{f} \mathcal{S}, Q\left(\left[u_{1}, u_{2}\right] \times\left[v_{1}, v_{2}\right]\right) \geqslant 0$. By the compactness of $\mathcal{S}$, there exist $\left(u_{1}^{*}, v_{2}^{*}\right) \in \mathcal{S}$ and $\left(u_{2}^{*}, v_{1}^{*}\right) \in \mathcal{S}$, such that

$$
\left\{\begin{array}{l}
f^{\mathcal{S}, Q}\left(u_{1}, v_{2}\right)=Q\left(u_{1}^{*}, v_{2}^{*}\right)-\left(u_{1}^{*}-u_{1}\right)^{+}-\left(v_{2}^{*}-v_{2}\right)^{+}, \\
f^{\mathcal{S}, Q}\left(u_{2}, v_{1}\right)=Q\left(u_{2}^{*}, v_{1}^{*}\right)-\left(u_{2}^{*}-u_{2}\right)^{+}-\left(v_{1}^{*}-v_{1}\right)^{+}
\end{array} .\right.
$$

For convenience, two cases are considered depending on whether $\left\{\left(u_{1}^{*}, v_{2}^{*}\right),\left(u_{2}^{*}, v_{1}^{*}\right)\right\}$ forms a non-decreasing set or a decreasing set.

Case 1: $\left(u_{1}^{*}, v_{2}^{*}\right)$ and $\left(u_{2}^{*}, v_{1}^{*}\right)$ form a non-decreasing set. From the definition (4) of $f^{\mathcal{S}, Q}$, it is clear that

$$
\left\{\begin{array}{l}
f^{\mathcal{S}, Q}\left(u_{1}, v_{1}\right) \geqslant Q\left(u_{2}^{*}, v_{1}^{*}\right)-\left(u_{2}^{*}-u_{1}\right)^{+}-\left(v_{1}^{*}-v_{1}\right)^{+} \\
f^{\mathcal{S}, Q}\left(u_{2}, v_{2}\right) \geqslant Q\left(u_{1}^{*}, v_{2}^{*}\right)-\left(u_{1}^{*}-u_{2}\right)^{+}-\left(v_{2}^{*}-v_{2}\right)^{+}
\end{array}\right.
$$

Using (5) and (6), the volume of the rectangle $\left[u_{1}, u_{2}\right] \times\left[v_{1}, v_{2}\right]$ can be bounded below as follows:

$$
\begin{aligned}
& f^{\mathcal{S}, Q}\left(u_{2}, v_{2}\right)-f^{\mathcal{S}, Q}\left(u_{1}, v_{2}\right)-f^{\mathcal{S}, Q}\left(u_{2}, v_{1}\right)+f^{\mathcal{S}, Q}\left(u_{1}, v_{1}\right) \\
& \geqslant {\left[Q\left(u_{1}^{*}, v_{2}^{*}\right)-\left(u_{1}^{*}-u_{2}\right)^{+}-\left(v_{2}^{*}-v_{2}\right)^{+}\right]-\left[Q\left(u_{1}^{*}, v_{2}^{*}\right)-\left(u_{1}^{*}-u_{1}\right)^{+}-\left(v_{2}^{*}-v_{2}\right)^{+}\right] } \\
& \quad-\left[Q\left(u_{2}^{*}, v_{1}^{*}\right)-\left(u_{2}^{*}-u_{2}\right)^{+}-\left(v_{1}^{*}-v_{1}\right)^{+}\right]+\left[Q\left(u_{2}^{*}, v_{1}^{*}\right)-\left(u_{2}^{*}-u_{1}\right)^{+}-\left(v_{1}^{*}-v_{1}\right)^{+}\right] \\
& \quad=\left[\left(u_{1}^{*}-u_{1}\right)^{+}-\left(u_{1}^{*}-u_{2}\right)^{+}\right]-\left[\left(u_{2}^{*}-u_{1}\right)^{+}-\left(u_{2}^{*}-u_{2}\right)^{+}\right] .
\end{aligned}
$$

It is clear that if $u_{1}^{*} \geqslant u_{2}^{*}$, then $V_{f} s, Q\left(\left[u_{1}, u_{2}\right] \times\left[v_{1}, v_{2}\right]\right) \geqslant 0$ using (7). On the other hand, consider when $u_{1}^{*}<u_{2}^{*}$; the process is similar. Indeed, realize that the following inequalities also hold:

$$
\left\{\begin{array}{l}
f^{\mathcal{S}, Q}\left(u_{1}, v_{1}\right) \geqslant Q\left(u_{1}^{*}, v_{2}^{*}\right)-\left(u_{1}^{*}-u_{1}\right)^{+}-\left(v_{2}^{*}-v_{1}\right)^{+}, \\
f^{\mathcal{S}, Q}\left(u_{2}, v_{2}\right) \geqslant Q\left(u_{2}^{*}, v_{1}^{*}\right)-\left(u_{2}^{*}-u_{2}\right)^{+}-\left(v_{1}^{*}-v_{2}\right)^{+}
\end{array}\right.
$$

and therefore using (5), it follows that,

$$
\begin{aligned}
& f^{\mathcal{S}, Q}\left(u_{2}, v_{2}\right)-f^{\mathcal{S}, Q}\left(u_{1}, v_{2}\right)-f^{\mathcal{S}, Q}\left(u_{2}, v_{1}\right)+f^{\mathcal{S}, Q}\left(u_{1}, v_{1}\right) \\
& \quad \geqslant\left[\left(v_{2}^{*}-v_{2}\right)^{+}-\left(v_{2}^{*}-v_{1}\right)^{+}\right]-\left[\left(v_{1}^{*}-v_{2}\right)^{+}-\left(v_{1}^{*}-v_{1}\right)^{+}\right] \geqslant 0,
\end{aligned}
$$

which follows from the fact that $v_{2}^{*} \leqslant v_{1}^{*}$, since $\left(u_{1}^{*}, v_{2}^{*}\right)$ and $\left(u_{2}^{*}, v_{1}^{*}\right)$ form a non-decreasing set, and we have assumed that $u_{1}^{*}<u_{2}^{*}$.

Case 2: Assume that $\left(u_{1}^{*}, v_{2}^{*}\right)$ and $\left(u_{2}^{*}, v_{1}^{*}\right)$ form a decreasing set. It is also known that $\left(u_{1}^{*}, v_{1}^{*}\right) \in \mathcal{S}$ and $\left(u_{2}^{*}, v_{2}^{*}\right) \in \mathcal{S}$. From the definition (4) of $f^{\mathcal{S}, Q}$, bound $f^{\mathcal{S}, Q}\left(u_{1}, v_{1}\right)$ and $f^{\mathcal{S}, Q}\left(u_{2}, v_{2}\right)$ from below:

$$
\left\{\begin{array}{l}
f^{\mathcal{S}, Q}\left(u_{1}, v_{1}\right) \geqslant Q\left(u_{1}^{*}, v_{1}^{*}\right)-\left(u_{1}^{*}-u_{1}\right)^{+}-\left(v_{1}^{*}-v_{1}\right)^{+} \\
f^{\mathcal{S}, Q}\left(u_{2}, v_{2}\right) \geqslant Q\left(u_{2}^{*}, v_{2}^{*}\right)-\left(u_{2}^{*}-u_{2}\right)^{+}-\left(v_{2}^{*}-v_{2}\right)^{+}
\end{array}\right.
$$

Writing down the volume of $f^{\mathcal{S}, Q}$ and using the aforementioned lower bounds on $f^{\mathcal{S}, Q}\left(u_{1}, v_{1}\right)$ and $f^{\mathcal{S}, Q}\left(u_{2}, v_{2}\right)$ it follows that the volume of the rectangle $\left[u_{1}, u_{2}\right] \times\left[v_{1}, v_{2}\right]$ is non-negative.

$$
\begin{aligned}
& f^{\mathcal{S}, Q}\left(u_{2}, v_{2}\right)-f^{\mathcal{S}, Q}\left(u_{1}, v_{2}\right)-f^{\mathcal{S}, Q}\left(u_{2}, v_{1}\right)+f^{\mathcal{S}, Q}\left(u_{1}, v_{1}\right) \\
& \geqslant {\left[Q\left(u_{2}^{*}, v_{2}^{*}\right)-\left(u_{2}^{*}-u_{2}\right)^{+}-\left(v_{2}^{*}-v_{2}\right)^{+}\right]-\left[Q\left(u_{1}^{*}, v_{2}^{*}\right)-\left(u_{1}^{*}-u_{1}\right)^{+}-\left(v_{2}^{*}-v_{2}\right)^{+}\right] } \\
& \quad-\left[Q\left(u_{2}^{*}, v_{1}^{*}\right)-\left(u_{2}^{*}-u_{2}\right)^{+}-\left(v_{1}^{*}-v_{1}\right)^{+}\right]+\left[Q\left(u_{1}^{*}, v_{1}^{*}\right)-\left(u_{1}^{*}-u_{1}\right)^{+}-\left(v_{1}^{*}-v_{1}\right)^{+}\right] \\
& \quad=Q\left(u_{2}^{*}, v_{2}^{*}\right)-Q\left(u_{1}^{*}, v_{2}^{*}\right)-Q\left(u_{2}^{*}, v_{1}^{*}\right)+Q\left(u_{1}^{*}, v_{1}^{*}\right) \geqslant 0,
\end{aligned}
$$


where the last inequality follows from the fact that $Q$ is 2 -increasing over $\mathcal{S}$ and $\left\{\left(u_{1}^{*}, v_{2}^{*}\right),\left(u_{2}^{*}, v_{1}^{*}\right)\right\}$ is a decreasing set. This completes the proof of Case 2.

Combining Case 1 and Case 2, it follows that $f^{\mathcal{S}, Q}$ is 2 -increasing. Since $f^{\mathcal{S}, Q}$ is Lipschitz, non-decreasing in each argument, and 2-increasing, Lemma 1 implies that the quasi-copula $B^{\mathcal{S}, Q}$ is 2-increasing in $(u, v)$ and therefore, by definition, a copula ${ }^{4}$.

As in the proof of Theorem 1 from Tankov(2011), note that

$$
A^{\mathcal{S}, Q}(u, v)=u-B^{\overline{\mathcal{S}}, \bar{Q}}(u, 1-v)=\overline{B^{\bar{S}}, \bar{Q}}(u, v)
$$

where $\overline{\mathcal{S}}$ is defined as $\overline{\mathcal{S}}=\{(a, b) \mid(a, 1-b) \in \mathcal{S}\}$ and $\bar{Q}(u, v)=u-Q(u, 1-v)$.

It is clear that $\overline{\mathcal{S}}$ satisfies condition (2) in Theorem 1 as $\mathcal{S}$ satisfies (2). In addition, $\bar{Q}$ is a quasi-copula which satisfies condition (3), since for $\forall\left(a_{0}, b_{0}\right),\left(a_{1}, b_{1}\right) \in \overline{\mathcal{S}}$ with $a_{0}<a_{1}, b_{0}<b_{1}$,

$$
\begin{aligned}
& \bar{Q}\left(a_{1}, b_{1}\right)+\bar{Q}\left(a_{0}, b_{0}\right)-\bar{Q}\left(a_{0}, b_{1}\right)-\bar{Q}\left(a_{1}, b_{0}\right) \\
& =a_{1}-Q\left(a_{1}, 1-b_{1}\right)+a_{0}-Q\left(a_{0}, 1-b_{0}\right)-\left(a_{0}-Q\left(a_{0}, 1-b_{1}\right)\right)-\left(a_{1}-Q\left(a_{1}, 1-b_{0}\right)\right) \\
& =Q\left(a_{0}, 1-b_{1}\right)+Q\left(a_{1}, 1-b_{0}\right)-Q\left(a_{0}, 1-b_{0}\right)-Q\left(a_{1}, 1-b_{1}\right) \geqslant 0,
\end{aligned}
$$

Using the proof that $B^{\mathcal{S}, Q}$ is a copula, it follows directly that $B^{\overline{\mathcal{S}}, \bar{Q}}$ is also a copula. Thus $\overline{B^{\overline{\mathcal{S}}, \bar{Q}}}$ is a copula and hence $A^{\mathcal{S}, Q}$ is a copula.

Theorem 2 gives alternative conditions than Tankov [25] and Bernard, Jiang and Vanduffel [3]. For example it can be applied with the compact $\mathcal{S}$ which consists of 4 points: $\mathcal{S}=\left\{\left(a_{0}, b_{0}\right),\left(a_{1}, b_{0}\right),\left(a_{0}, b_{1}\right),\left(a_{1}, b_{1}\right)\right\}$ for some $0 \leqslant a_{0}<a_{1} \leqslant 1$ and $0 \leqslant b_{0}<b_{1} \leqslant 1$. This compact set does not satisfy the monotonicity property needed in Tankov [25] and does not satisfy the connectivity property of Bernard et al. [3]. However as soon as $Q$ satisfies condition (3) (which is automatically satisfied when $Q$ is a copula), both $A^{\mathcal{S}, Q}$ and $B^{\mathcal{S}, Q}$ are copulas. Note that it is also possible to construct a compact, e.g. $\mathcal{S}_{1}:=\left\{y=2 x, x \in\left[\frac{1}{4} \frac{1}{3}\right]\right\}$, that satisfies conditions in Bernard, Jiang and Vanduffel [3] for $B^{\mathcal{S}_{1}, Q}$ to be a copula but not (2) in Theorem 2. Note however that the condition for $A^{\mathcal{S}_{1}, Q}$ to be a copula is not satisfied as $\mathcal{S}_{1}$ is an increasing set and thus the two bounds are not simultaneously copulas.

There are many other sets satisfying the assumptions of Theorem 2 including for example two vertical areas $\{x \in$ $\left.\left[\frac{1}{4}, \frac{1}{3}\right], x \in\left[\frac{2}{3}, \frac{3}{4}\right], y \in[0,1]\right\}$ (or more vertical areas), similarly a set of horizontal areas or unions of horizontal and vertical areas satisfy (2).

Note that when $\mathcal{S}$ is a rectangle that intersects the boundaries of $[0,1]^{2}$ then (3) is automatically satisfied in view of Section 4 in Genest et al. [12].

\section{Application to Quantitative Risk Management}

We here describe potential applications of Theorem 2 in quantifying uncertainty on dependence (which is a type of model risk when marginals can be well estimated but when the dependence may be misspecified). See Embrechts et al. [10] for a discussion of quantifying model risk by computing bounds on Value-at-Risk when marginals are given and dependence is totally unspecified. The goal of this section is to illustrate a methodology to assess model risk and to illustrate the potential use of bounds on copulas.

Consider a bivariate risk $(X, Y)$. We assume that one has an accurate assessment of the marginal distributions of $X$ and $Y$ but only partial information on the dependence. Here are some potential applications.

For example, consider an insurance company with two lines of business: life insurance and non-life insurance. Let us denote the respective aggregate losses of each business line as $X$ and $Y$. We assume that the risk of the company can be assessed as $E[h(X, Y)]$ where $h$ is some bivariate risk measure. For example $h(X, Y)=(\max (X, Y)-K)^{+}$for some $K \geqslant 0$, or $h(X, Y):=(X+Y-K)^{+}$. These risk measures are particularly relevant to a reinsurer. The first risk measure

${ }^{4}$ Note that Theorem 7.1 of Durante et al. [9] can also be invoked here to conclude that $B^{\mathcal{S}, Q}$ is a copula. 
is indeed the pure premium (expected value) for a stop-loss policy written on the maximum loss of this company. The second risk measure is the pure premium for a stop-loss on the aggregate risk of the company.

In addition, we assume that the copula between $X$ and $Y$ is state-dependent, that is, it may change when market conditions change. Specifically, we assume that both risks are observed for many years in "normal" conditions and that very few observations are available during market crashes and catastrophes (tail dependence is hard to observe). We thus suppose that the dependence observed in the data is essentially well suited for $X$ and $Y$ each belonging to the $90 \%$ confidence intervals $X \in\left[a_{0}, a_{1}\right]$ and $Y \in\left[b_{0}, b_{1}\right]$ and that the copula is unspecified outside of the $90 \%$ confidence intervals as there is not enough data.

Another example is to study a bivariate derivative linked to two stocks or two sectors. The respective losses of the two underlying stocks (or sector indices) may look independent most of the time. However when the market is stressed, both companies (or sectors) can be hit by common shocks, so that the two companies become dependent. Such systemic elements are not observed in normal conditions. Pricing this bivariate derivative by ignoring this possible change of dependence in extreme scenarios would potentially underestimate or overestimate its price.

It may also be useful to specify the dependence in the tails only. For example, one may choose to not specify the dependence in the middle of the distribution and constrain it to being comonotonic or antimonotonic when one knows that the two companies will be hit the same way when the market is in extreme conditions (in extremely good and in extremely bad scenarios). This is also referred as systemic risk factors. The set of constraints on the dependence then consists of four rectangles corresponding to the four corners of the unit square. Our numerical example illustrates these different situations.

\subsection{Setting}

We propose to model the problem as follows. The first case that we consider is $\mathcal{S}=\left[a_{0}, a_{1}\right] \times\left[b_{0}, b_{1}\right]$. Assume that the dependence between the risks $X$ and $Y$ is known on $\mathcal{S}$ and modeled by a copula $Q$ over the region $\mathcal{S}=\left[a_{0}, a_{1}\right] \times\left[b_{0}, b_{1}\right]$ (Figure 1, Case 1). Similarly we define $\mathcal{S}$ as the union of the four corners as can be seen from Case 2 in Figure 1.

$$
\forall x \in\left[a_{0}, a_{1}\right], \forall y \in\left[b_{0}, b_{1}\right], P(X \leqslant x, Y \leqslant y)=Q\left(F_{X}(x), F_{Y}(y)\right)
$$

In practice, $Q$ could be obtained from fitting from market data, where there is sufficient data available for a good fit of a copula.
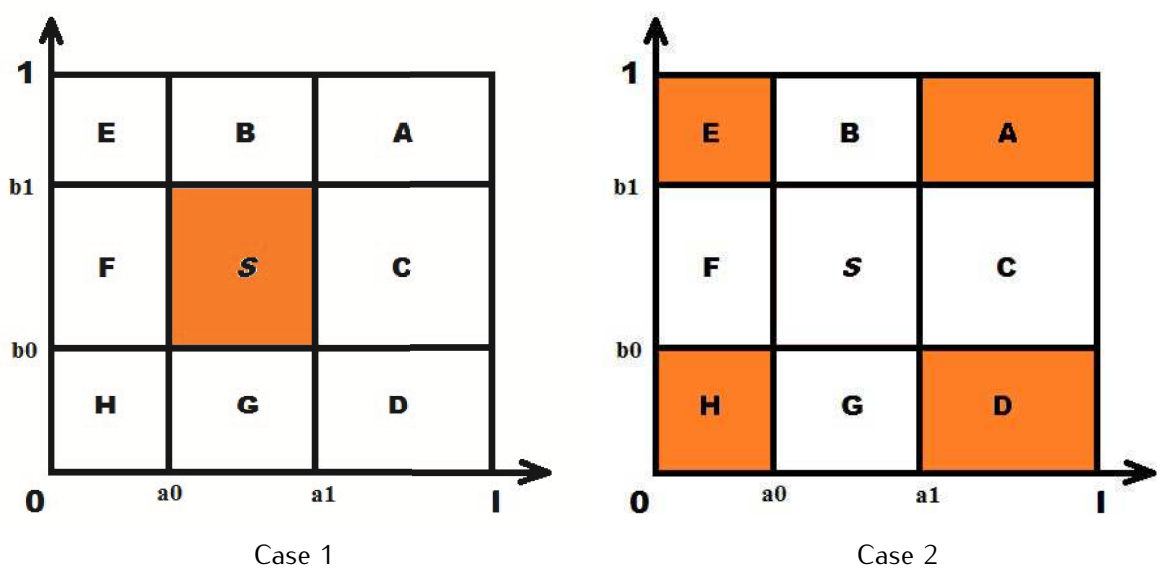

Fig 1. Partition of the unit square. In each case, the darker areas denote the compact where the copula is known and equal to $Q$ as formulated in (9).

We are now solving for the minimum and maximum copulas so that we could use these results to find bounds on prices or capital requirements. Consider the bivariate risk measure $E[h(X, Y)]$ where $h(X, Y)=(\max (X, Y)-K)^{+}$and assume the marginals of $X$ and $Y$ are known. For instance we assume that both risks $X$ and $Y$ are lognormally distributed so 
that $F_{X}(x)=\Phi\left\{\frac{\ln (x)-\mu_{x}}{\sigma_{X}}\right\}$ and $F_{Y}(y)=\Phi\left\{\frac{\ln (y)-\mu_{y}}{\sigma_{y}}\right\}$.

$$
P(X \leqslant x, Y \leqslant y)=C\left(F_{X}(x), F_{Y}(y)\right)=Q\left(\Phi\left\{\frac{\ln (x)-\mu_{x}}{\sigma_{x}}\right\}, \Phi\left\{\frac{\ln (y)-\mu_{y}}{\sigma_{y}}\right\}\right)
$$

for all $(x, y) \in \mathcal{S}$ and where $C$ is the copula for $(X, Y)$ and $C=Q$ on $\mathcal{S}$. To illustrate the study we consider $Q(u, v)=u v$ (independent risk), $Q(u, v)=\min (u, v)$ (comonotonic risks) and $Q(u, v)=\max (u+v-1,0)$ (anti monotonic risks) in the example but our analysis holds for more general choice of $Q$.

\subsection{Theoretical Developments}

Let us define $\pi(C)=\mathbb{E}[h(X, Y)]$ where $C$ is the copula for $(X, Y)$ and observe that

$$
\pi(C)=\int_{0}^{\infty} \int_{0}^{\infty} h(x, y) d C\left(F_{X}(x), F_{Y}(y)\right)
$$

Tchen [26] showed that for every 2-increasing function $h$ such that (10) exists, the mapping $C \mapsto \pi(C)$ is non-decreasing with respect to the concordance order of copulas (for example for $\left.h(X, Y)=(X+Y-K)^{+}\right)$. Similar properties can be proved for 2-decreasing functions. For example, since $h(X, Y)=(\max (X, Y)-K)^{+}$is 2-decreasing, and $A^{\mathcal{S}, Q}, B^{\mathcal{S}, Q}$ and $C$ are quasi-copulas such that for all $(u, v) \in[0,1]^{2}, B^{\mathcal{S}, Q}(u, v) \leqslant C(u, v) \leqslant A^{\mathcal{S}, Q}(u, v)$, we have

$$
\pi_{A}:=\pi\left(A^{\mathcal{S}, Q}\right) \leqslant \pi(C) \leqslant \pi_{B}:=\pi\left(B^{\mathcal{S}, Q}\right) .
$$

As $\mathcal{S}$ is a compact set and $Q$ is a copula, it satisfies all of the properties from Theorem 2 , then $A^{\mathcal{S}, Q}(u, v)$ and $B^{\mathcal{S}, Q}(u, v)$ are copulas. Since both $A^{\mathcal{S}, Q}$ and $B^{\mathcal{S}, Q}$ are copulas, the bounds $\pi_{B}$ and $\pi_{A}$ are attained and correspond to a possible dependence between $X$ and $Y$.

For the purposes of this example, it is more convenient to express (10) as follows:

$$
\pi(C)=\int_{0}^{1} \int_{0}^{1} h\left(F_{X}^{-1}(u), F_{Y}^{-1}(v)\right) d C(u, v)
$$

Similarly as in Nelsen [18], page 38, the double-integral $\pi_{A}$ (resp. $\pi_{B}$ ) can be rewritten as integrals over the support of $A^{\mathcal{S}, Q}$ (resp. $B^{\mathcal{S}, Q}$ ). Let us give some explanations to compute $\pi_{A}$. It is clear that $A^{\mathcal{S}, Q}$ is equal to $Q$ on $\mathcal{S}$ but is singular everywhere else.

In what follows, we assume $Q(u, v)=u v$ and $\mathcal{S}=\left[a_{0}, a_{1}\right] \times\left[b_{0}, b_{1}\right]$. The support of $A^{\mathcal{S}, Q}$ is displayed in the first column of Figure 2. To obtain Figure 2, we simulate 10,000 couples $(U, V)$ from the copula $A^{\mathcal{S}, Q}$ when $Q(u, v)=u v$ and $\mathcal{S}=\left[a_{0}, a_{1}\right] \times\left[b_{0}, b_{1}\right]$. The simulation procedure follows from a general method explained for instance in Mai and Sherer [16]. In our special case, we first compute $A^{\mathcal{S}, Q}(u, v)$ using (1) for all $(u, v) \in[0,1]^{2}$, and then calculate $A_{u}:=v \mapsto \frac{\partial}{\partial u}\left(A^{\mathcal{S}, Q}(u, v)\right)$. Recall that it is a conditional $\operatorname{cdf}(P(V \leqslant v \mid U=u))$, and we then compute its pseudo inverse $A_{u}^{-1}$ similarly defined as the inverse of a cdf. We obtain

$$
A_{u}^{-1}(w)=\left\{\begin{array}{cl}
u & \text { if } 0 \leqslant u \leqslant a_{0} b_{0} \\
\frac{u}{a_{0}} & \text { if } a_{0} b_{0}<u \leqslant a_{0} b_{1} \\
u+b_{1}-a_{0} b_{1} & \text { if } a_{0} b_{1}<u \leqslant a_{0} \\
k(u, w) & \text { if } a_{0}<u \leqslant a_{1} \\
u-a_{1}+a_{1} b_{0} & \text { if } a_{1}<u \leqslant a_{1}+b_{0}-a_{1} b_{0} \\
\frac{u-a_{1}}{1-a_{1}} & \text { if } a_{1}+b_{0}-a_{1} b_{0}<u \leqslant a_{1}+b_{1}-a_{1} b_{1} \\
u & \text { if } a_{1}+b_{1}-a_{1} b_{1}<u \leqslant 1
\end{array}\right.
$$

where $k(u, w):=b_{0} u \mathbb{1}_{w<b_{0}}+w \mathbb{1}_{b_{0}<w<b_{1}}+\left(b_{1}+\left(1-b_{1}\right) u\right) \mathbb{1}_{w \geqslant b_{1}}$. The algorithm to simulate $(U, V)$ consists of the two following steps. First we simulate two independent variables $(U, W)$ from the uniform distribution $\mathcal{U}(0,1)$. Second, we compute $V=A_{U}^{-1}(W)$. We then repeat these two steps 10,000 times and display the couples $(U, V)$ in Figure 2. 

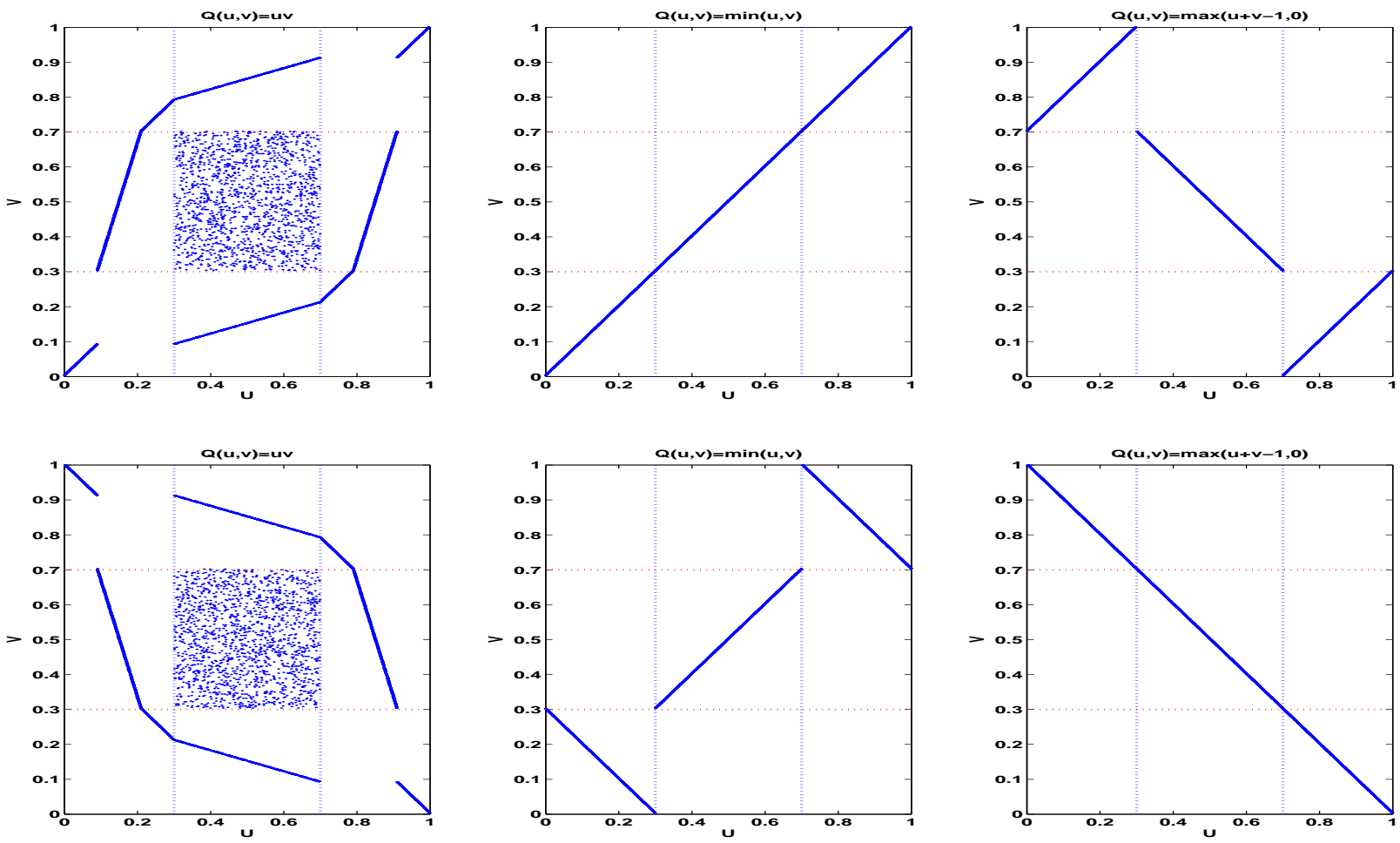

Fig 2. Case 1 for $\mathcal{S}$ in Figure 1. Supports of $A^{\mathcal{S}, Q}$ (top graphs) and $B^{\mathcal{S}, Q}$ (bottom graphs) when $Q(u, v)=u v, Q(u, v)=\min (u, v)$ or $Q(u, v)=$ $\max (u+v-1)$ and the copula is constrained on a rectangle $a_{0}=0.3, b_{0}=0.3, a_{1}=0.7, b_{1}=0.7$.

Let $\Gamma$, denote the support of $A^{\mathcal{S}, Q}$ over a particular subset $J \subset[0,1]^{2}$. Over region $\mathbf{H}=\left\{\mathbf{u} \leqslant \mathbf{a}_{0}, \mathbf{v} \leqslant \mathbf{b}_{0}\right\}$ (see Figure 1 , Case 1 for a graphical illustration), the support of $A^{\mathcal{S}, Q}$ is given by

$$
\Gamma_{H}:=\left\{(u, v) \in H \mid u=v: u \leqslant Q\left(a_{0}, b_{0}\right), v \leqslant Q\left(a_{0}, b_{0}\right)\right\}
$$

Similarly on region $A=\left\{\mathbf{u} \geqslant \mathbf{a}_{1}, \mathbf{v} \geqslant \mathbf{b}_{1}\right\}$,

$$
\Gamma_{A}:=\left\{(u, v) \in A \mid u=v: u \geqslant a_{1}+b_{1}-Q\left(a_{1}, b_{1}\right), v \geqslant a_{1}+b_{1}-Q\left(a_{1}, b_{1}\right)\right\}
$$

A similar analysis over each region yields the following supports: $\Gamma_{G}:=\left\{(u, v) \in G \mid v=Q\left(u, b_{0}\right)\right\} ; \Gamma_{D}:=\{(u, v) \in$ $\left.D \mid v=u-a_{1}+Q\left(a_{1}, b_{0}\right)\right\} ; \Gamma_{C}:=\left\{(u, v) \in C \mid v-Q\left(a_{1}, v\right)=u-a_{1}\right\} ; \Gamma_{F}:=\left\{(u, v) \in F \mid Q\left(a_{0}, v\right)=u\right\} ; \Gamma_{E}:=$ $\left\{(u, v) \in E \mid v=u+b_{1}-Q\left(a_{0}, b_{1}\right)\right\} ;$ and $\Gamma_{B}:=\left\{(u, v) \in B \mid v=u-Q\left(u, b_{1}\right)+b_{1}\right\}$.

Denote by $\Gamma=\bigcup_{i \in \mathcal{I}} \Gamma_{i}$ where $\mathcal{I}=\{A, B, C, D, E, F, G, H\}$ and simplify the double integral $\pi_{A}$ by splitting the computation into nine parts. The only region we have ignored so far is $\mathcal{S}$, where the support of $A^{\mathcal{S}, Q}$ is equal to the support of the non-degenerate quasi-copula $Q$ restricted to $\mathcal{S}$ as $A^{\mathcal{S}, Q}=Q$. For example when $Q(u, v)=u v$ then $\Gamma_{\mathcal{S}}=\mathcal{S}$. The double integral $\pi_{A}$ can be reduced to single integrals over $A, B, C, D, E, F, G, H$ and a double integral over $\mathcal{S}$. The calculations are straightforward (see Appendix A for more details). Similar calculations can be done with other choices of $Q(u, v)$ and to obtain $\pi_{B}$.

\subsection{Numerical Analysis}

Assume $\mu_{x}=\mu_{y}=\mu=2, a_{1}=b_{1}, a_{0}=b_{0}$, that $\sigma_{x}=\sigma_{y}=\sigma=1$ and that $K=5$. We assume that $q_{\alpha}$ and $q_{1-\alpha}$ correspond respectively to the quantile at the level $\alpha$ and $1-\alpha$ where $\alpha$ takes different values. Note that 

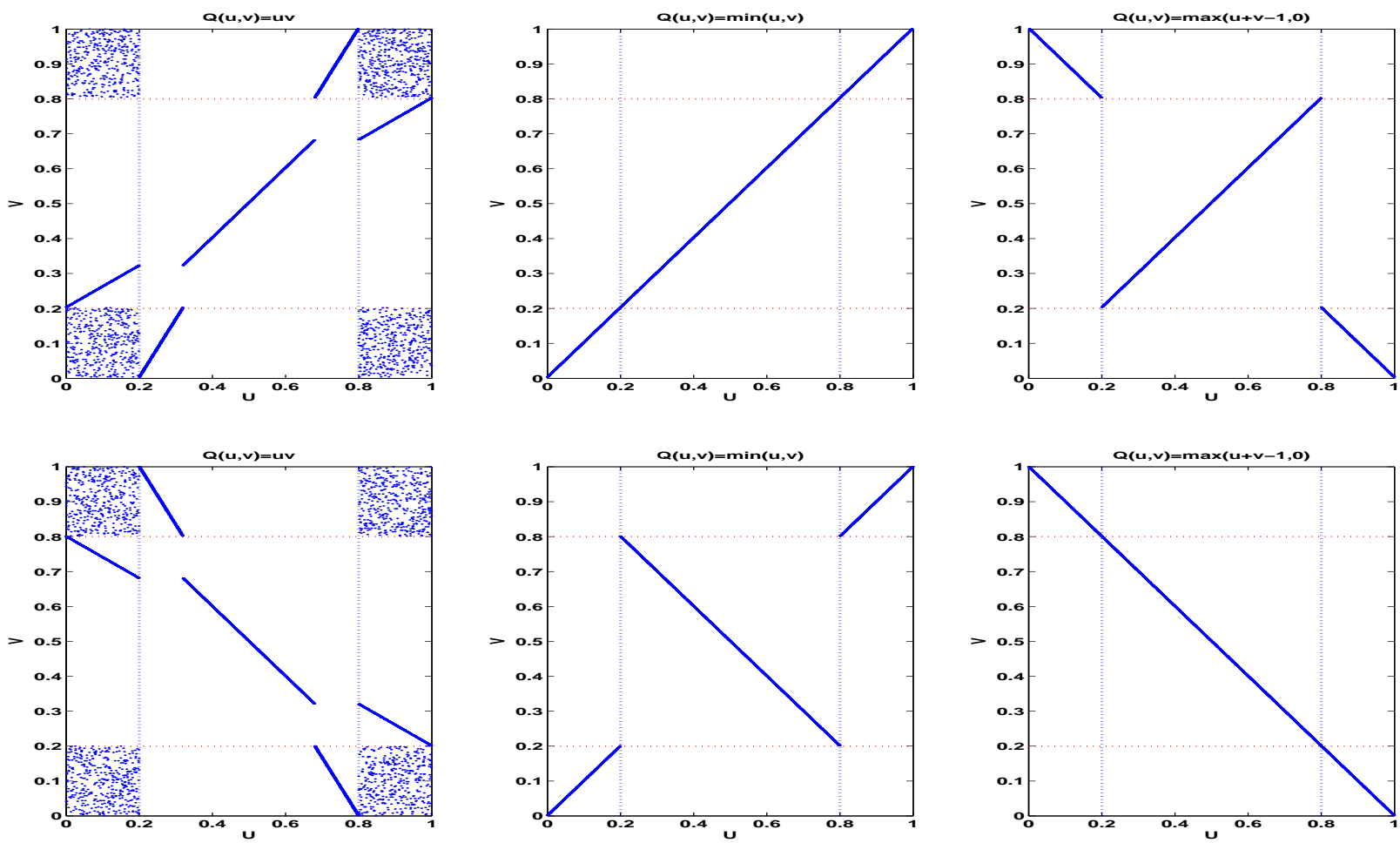

Fig 3. Case 2 for $\mathcal{S}$ in Figure 1. Supports of $A^{\mathcal{S}, Q}$ (top graphs) and $B^{\mathcal{S}, Q}$ (bottom graphs) when $Q(u, v)=u v, Q(u, v)=\min (u, v)$ or $Q(u, v)=$ $\max (u+v-1)$ and the copula is constrained in the 4 corners of the unit square where $a_{0}=0.3, b_{0}=0.3, a_{1}=0.7, b_{1}=0.7$.

Table 1. Numerical example $a_{0}=b_{0}=\alpha$ and $a_{1}=b_{1}=1-\alpha$. We compute $\pi_{A}$ and $\pi_{B}$ which are the respective upper and lower bounds of $E[h(X, Y)]$ when $C(u, v)=u v, C(u, v)=\min (u, v)$ and $C(u, v)=\max (0, u+v-1)$ for the extreme cases $\alpha=0$ and $\alpha=1 / 2$. All numbers are obtained with a standard deviation smaller than 0.01 .

\begin{tabular}{c||c|c||c|c||c|c||}
\multicolumn{1}{c||}{$Q(u, v)$} & \multicolumn{2}{c||}{$u v$} & \multicolumn{2}{c||}{$\min (u, v)$} & \multicolumn{2}{c||}{$\max (0, u+v-1)$} \\
\hline & Case 1 & Case 2 & Case 1 & Case 2 & Case 1 & Case 2 \\
\hline \multirow{2}{*}{$\alpha=0$} & $\pi_{B}=13.7$ & $\pi_{B}=15.5$ & $\pi_{B}=7.9$ & $\pi_{B}=15.5$ & $\pi_{B}=15.5$ & $\pi_{B}=15.5$ \\
& $\pi_{A}=13.7$ & $\pi_{A}=7.9$ & $\pi_{A}=7.9$ & $\pi_{A}=7.9$ & $\pi_{A}=15.5$ & $\pi_{A}=7.9$ \\
\hline \multirow{2}{*}{$\alpha=\frac{1}{2}$} & $\pi_{B}=14.9$ & $\pi_{B}=13.7$ & $\pi_{B}=13.1$ & $\pi_{B}=7.9$ & $\pi_{B}=15.5$ & $\pi_{B}=15.5$ \\
& $\pi_{A}=9.10$ & $\pi_{A}=13.7$ & $\pi_{A}=7.9$ & $\pi_{A}=7.9$ & $\pi_{A}=15.49$ & $\pi_{A}=15.5$ \\
\hline
\end{tabular}

$a_{0}=F_{X}\left(q_{\alpha}\right)=\alpha$ and $a_{1}=F_{X}\left(q_{1-\alpha}\right)=1-\alpha$. Similarly $b_{0}=\alpha$ and $b_{1}=1-\alpha$. Figure 4 and Table 1 show the impact on the quantity $\pi(C)$ of the model risk on the copula.

Note that in Case 1, when $\alpha=0$ and in Case 2, when $\alpha=1 / 2$ the copula is fully fixed and $\pi_{A}=\pi_{B}$. In Case 1 , when $\alpha=$ $1 / 2$, the copula is fixed at one point and we find back the results of Nelsen [18], page 71, section 3.2, Figure 3.10 on bounds on copulas when there is a constraint at one point. When $\alpha=1 / 2$ and $Q(u, v)=\max (0, u+v-1)$, then the constraint on the copula becomes $C(1 / 2,1 / 2)=0$. Obviously $B(u, v)=\max (0, u+v-1)$ and $A(u, v)=\min \left(u, v,(u-1 / 2)^{+}+(v-1 / 2)^{+}\right)$. This implies that $A(u, v)=B(u, v)$ for all $(u, v) \in\{[0,1 / 2] \times[0,1 / 2]\} \cup\{[1 / 2,1] \times[1 / 2,1]\}$. The constraint at 1 point is already quite constrictive, and it turns out that there is almostno difference between $\pi_{A}$ and $\pi_{B}$ for a constraint of 1 point only and thus for all $\alpha \in[0,1 / 2]$. Finally for Case 2 , when $\alpha=0$, the problem seems to be constrained in the 4 corners, but the constraints are automatically satisfied and thus we find back the Fréchet bounds.

The limit cases obtained in Table 1 appear now clearly on the graph 4 obtained by Monte Carlo simulations representing $\pi_{A}$ and $\pi_{B}$ in Case 1 and Case 2 for the 3 types of constraints considered above. 


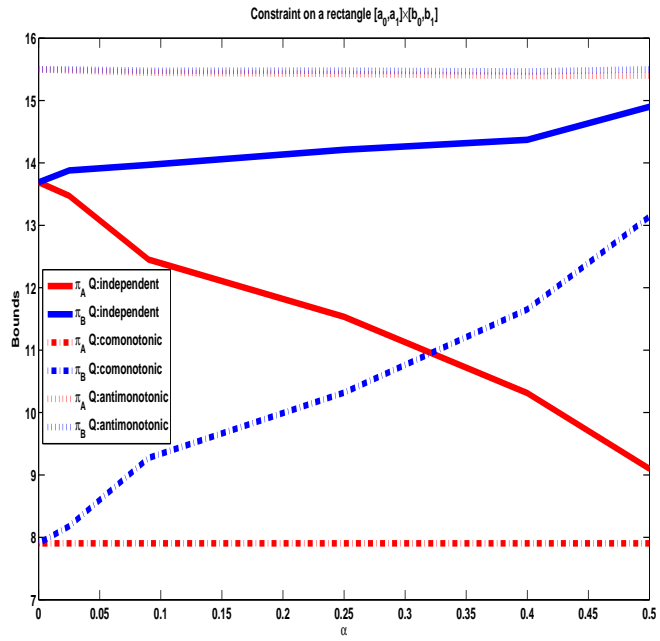

Case 1

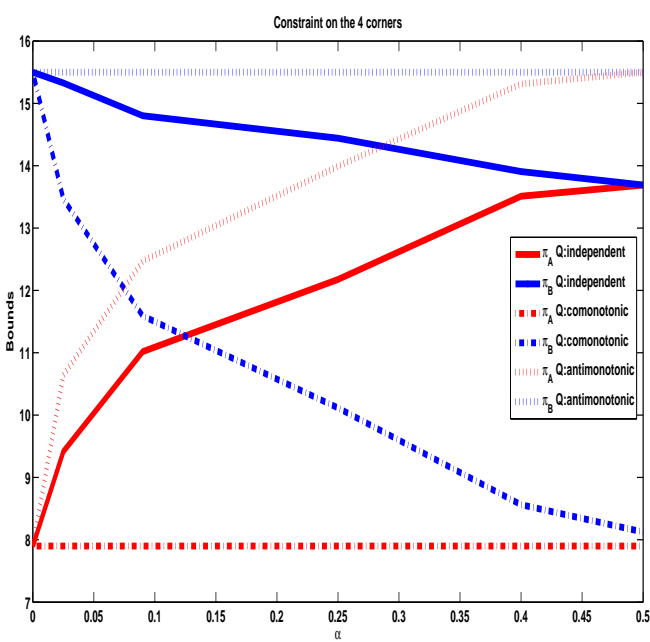

Case 2

Fig 4. $\pi_{A}$ and $\pi_{B}$ with respect to $\alpha$ when the information on the copula is only known over the square $[\alpha, 1-\alpha]^{2}$. On each graph, we show the upper bound and the lower bound for the comonotonic, antimonotonic and independence copula.

\section{Compact set $\mathcal{S}$ with 2 points}

In this last section we assume that there are exactly two constraints on the copula. Nelsen [18] derives best possible bounds when there is one constraint but the problem is more difficult when there is more than one constraint as either the lower bound or the upper bound may not be a copula. Thus the bounds are not sharp. The purpose of this section is to illustrate how to construct the best bounds in the sense of the infinite norm in the case when there are two constraints. Sadooghi-Alvandi et al. [23] also solve this problem but assume that the two constraints are either non-decreasing or non-increasing and thus are special cases of Tankov [25] main result. ${ }^{5}$ Hereafter, we derive explicit expressions for the smallest copula above the lower bound and the largest copula below the upper bound for the infinite norm. To do so, we start with a lemma. Note that although the derivations are done with two constraints, similar derivations can be done with a given number of constraints but this will lead to tedious calculations.

\section{Lemma 3.}

The infimum of functions with the 1-Lipschitz property also is 1-Lipschitz property.

We omit the proof of this lemma as it follows from the general fact that the aggregation of Lipschitz functions preserves the Lipschitz property of kernel aggregation functions (in particular max and min) are used, see for instance Grabish et al. [13] and Kolesárová et al. [15].

\section{Theorem 4.}

Let $\mathcal{S}=\left\{\left(a_{0}, b_{0}\right),\left(a_{1}, b_{1}\right)\right\}$ be an increasing set $\left(a_{0}<a_{1}, b_{0}<b_{1}\right)$ and $Q$ be a quasi-copula, such that $Q\left(a_{0}, b_{0}\right)=$

5 Precisely, Tankov [25] proves that if $\mathcal{S}$ is increasing, then $B^{\mathcal{S}, Q}$ is a copula and if $\mathcal{S}$ is decreasing, then $A^{\mathcal{S}, Q}$ is a copula. Thus, in Theorem 2.1 of Sadooghi-Alvandi et al. [23], $\mathcal{S}$ is increasing, $C_{n}$ is obviously not empty. Similarly, when $\mathcal{S}$ is decreasing which is indicated in Theorem 2.4 of Sadooghi-Alvandi et al. [23], $C_{n}$ is also not empty. Theorem 2.2 and 2.3 of Sadooghi-Alvandi et al. [23] assume that $\mathcal{S}$ is increasing or decreasing thus the same conclusion as in Tankov [25] is obtained. Although it seems at first that the result of Sadooghi-Alvandi et al. [23] is a special case of Tankov [25], the proofs are different. 
$\theta_{0}, Q\left(a_{1}, b_{1}\right)=\theta_{1}$. Let

$$
\mathcal{C}=\left\{C \text { copula } \mid C\left(a_{0}, b_{0}\right)=\theta_{0}, C\left(a_{1}, b_{1}\right)=\theta_{1}\right\}
$$

(i) $\exists C^{*} \in \mathcal{C}$ such that

$$
\left\|A^{\mathcal{S}, Q}-C^{*}\right\|_{\infty}=\inf _{C \in \mathcal{C}}\left\|A^{\mathcal{S}, Q}-C\right\|_{\infty}
$$

(ii) $C^{*}$ satisfies $C^{*}\left(a_{0}, b_{1}\right)=\alpha^{*}$ and $C^{*}\left(a_{1}, b_{0}\right)=\beta^{*}$, where $\alpha^{*}$ solves the following equality

$$
\min \left(a_{0}-\alpha, \theta_{0}-\alpha+b_{1}-b_{0}, \theta_{1}-\alpha\right)=\min \left(b_{0}+\alpha-\gamma, \theta_{0}+\alpha-\gamma+a_{1}-a_{0}, \theta_{1}+\alpha-\gamma\right),
$$

where $\gamma=\min \left\{\theta_{0}+\theta_{1}, \min \left(a_{0}, b_{1}\right)+\min \left(a_{1}, b_{0}\right)\right\}$ and $\beta^{*}=\gamma-\alpha^{*}$.

Proof. Tankov [25] shows that if $\left\{\left(a_{0}, b_{0}\right),\left(a_{1}, b_{1}\right)\right\}$ is an increasing set then $B^{\mathcal{S}, Q}$ is a copula; hence, $\mathcal{C}$ defined by (12) is non-empty. To establish the existence of $C^{*}$, we establish that $\mathcal{C}$ is closed and that $g: \mathcal{C} \rightarrow \mathbb{R}$, where $g(C)=\left\|A^{\mathcal{S}, Q}-C\right\|_{\infty}$ is continuous.

To prove that $\mathcal{C}$ is closed, consider a sequence $\left\{D_{n}\right\}$ in $\mathcal{C}$ converging uniformly to $D^{*}$. Since each $D_{n}$ is a copula, and uniform convergence preserves the properties of copulas, $D^{*}$ is a copula. Moreover, for constant $\theta_{0}$ and $\theta_{1}$ such that $D_{n}\left(a_{0}, b_{0}\right)=\theta_{0}$ and $D_{n}\left(a_{1}, b_{1}\right)=\theta_{1}$,

$$
\lim _{n \rightarrow \infty} D_{n}\left(a_{0}, b_{0}\right)=D^{*}\left(a_{0}, b_{0}\right)=\theta_{0} \text { and } \lim _{n \rightarrow \infty} D_{n}\left(a_{1}, b_{1}\right)=D^{*}\left(a_{1}, b_{1}\right)=\theta_{1} .
$$

Therefore, $D^{*} \in \mathcal{C}$ and hence $\mathcal{C}$ is closed. To prove the continuity of $g$, consider $C_{1} \in \mathcal{C}$ and $C_{2} \in \mathcal{C}$

$$
\left|g\left(C_{1}\right)-g\left(C_{2}\right)\right|=\left|\left\|A^{\mathcal{S}, Q}-C_{1}\right\|_{\infty}-\left\|A^{\mathcal{S}, Q}-C_{2}\right\|_{\infty}\right| \leqslant\left\|C_{1}-C_{2}\right\|_{\infty}
$$

where the inequality follows from the triangle inequality. Thus $g$ is 1 -Lipschitz and therefore continuous.

As $\mathcal{C}$ is closed and $g(C)=\left\|A^{\mathcal{S}, Q}-C\right\|_{\infty}$ is continuous, the infimum in (13) is a minimum. Let us denote this minimum by $C^{*}$. Define $\alpha^{*}:=C^{*}\left(a_{0}, b_{1}\right), \beta^{*}:=C^{*}\left(a_{1}, b_{0}\right)$ and $\widetilde{\mathcal{S}}$ by

$$
\widetilde{\mathcal{S}}=\left\{\left(a_{0}, b_{0}\right),\left(a_{0}, b_{1}\right),\left(a_{1}, b_{0}\right),\left(a_{1}, b_{1}\right)\right\} .
$$

Since $C^{*}$ is a copula, and $\widetilde{\mathcal{S}}$ satisfies condition (2). Using the result of Theorem $2, A^{\widetilde{\mathcal{S}}}, C^{*}$ is a copula $\widetilde{Q}$ such that $\widetilde{Q}\left(a_{0}, b_{0}\right)=\theta_{0}, \widetilde{Q}\left(a_{0}, b_{1}\right)=\alpha^{*}, \widetilde{Q}\left(a_{1}, b_{0}\right)=\beta^{*}$, and $\widetilde{Q}\left(a_{1}, b_{1}\right)=\theta_{1}$. We have that $C^{*} \leqslant A^{\tilde{\mathcal{S}}}, C^{*}$ by definition of $A^{\widetilde{\mathcal{S}}}, C^{*}$ as an upper bound on a set of copulas with constraints on $\widetilde{\mathcal{S}}$. We also have $A^{\widetilde{\mathcal{S}}, C^{*}} \leqslant A^{\mathcal{S}, C^{*}}=A^{\mathcal{S}, Q}$ because $C^{*}=Q$ on $\mathcal{S}$ and $\mathcal{S} \subset \widetilde{\mathcal{S}}$. Moreover $\left\|A^{\mathcal{S}, Q}-C^{*}\right\|_{\infty} \leqslant\left\|A^{\mathcal{S}, Q}-A^{\tilde{\mathcal{S}}}, C^{*}\right\|_{\infty}$ because of the optimality of $C^{*}$ in (13). Thus $C^{*}=A^{\widetilde{\mathcal{S}}} C^{*}$.

Let us now solve for $\alpha^{*}$ and $\beta^{*}$. To do so, we can simplify the initial problem by the following parametric optimization over all $\alpha$ and $\beta$ such that $C^{*}$ is a copula

$$
\left\|A^{\mathcal{S}, Q}-A^{\tilde{\mathcal{S}}, C^{*}}\right\|_{\infty}=\inf _{\alpha, \beta}\left\|A^{\mathcal{S}, Q}-A^{\tilde{\mathcal{S}}, \widetilde{Q}}\right\|_{\infty}
$$

where $\widetilde{Q}$ is defined previously with $\widetilde{Q}\left(a_{0}, b_{1}\right)=\alpha, \widetilde{Q}\left(a_{1}, b_{0}\right)=\beta, \widetilde{Q}\left(a_{0}, b_{0}\right)=\theta_{0}$ and $\widetilde{Q}\left(a_{1}, b_{1}\right)=\theta_{1}$.

Notice that $A^{\widetilde{\mathcal{S}}, \widetilde{Q}}(u, v)=\min \left(f_{\alpha}(u, v), f_{\beta}(u, v), A^{\mathcal{S}, Q}(u, v)\right)$, where $f_{\alpha}(u, v)=\alpha+\left(u-a_{0}\right)^{+}+\left(v-b_{1}\right)^{+}$and $f_{\beta}(u, v)=$ $\beta+\left(u-a_{1}\right)^{+}+\left(v-b_{0}\right)^{+}$. For convenience, the optimality can be written as follows:

$$
\min _{\alpha, \beta}\left\|A^{\mathcal{S}, Q}-A^{\tilde{\mathcal{S}}, \tilde{Q}}\right\|_{\infty}=\min _{\alpha, \beta}\left\{\max _{(u, v) \in[0,1]^{2}}\left\{A^{\mathcal{S}, Q}(u, v)-\min \left(f_{\alpha}(u, v), f_{\beta}(u, v), A^{\mathcal{S}, Q}(u, v)\right)\right\}\right\}
$$

For later reference, recall some key properties of copulas:

$$
\begin{aligned}
& \theta_{0} \leqslant \alpha, \beta \leqslant \theta_{1}, \quad \alpha+\beta \leqslant \theta_{0}+\theta_{1}, \\
& \max (u+v-1,0) \leqslant Q(u, v) \leqslant \min (u, v), \\
& Q\left(u_{2}, v_{2}\right)-Q\left(u_{1}, v_{1}\right) \leqslant\left(u_{2}-u_{1}\right)+\left(v_{2}-v_{1}\right) .
\end{aligned}
$$


For the purpose of this proof, it is more convenient to consider nine different cases, by dividing the domain $[0,1] \times[0,1]$ into nine areas as in Figure 1.

$$
\left\|A^{\mathcal{S}, Q}-A^{\tilde{\mathcal{S}}, \tilde{Q}}\right\|_{\infty}=\max _{(u, v) \in[0,1]^{2}}\left|A^{\mathcal{S}, Q}(u, v)-A^{\tilde{\mathcal{S}}, \tilde{Q}}(u, v)\right|=\max \left\{M_{A}, M_{B}, M_{C}, M_{D}, M_{E}, M_{F}, M_{G}, M_{H}, M_{\mathcal{S}}\right\}
$$

where $M_{\mathcal{I}}=\max _{(u, v) \in \mathcal{I}}\left|A^{\mathcal{S}, Q}(u, v)-A^{\tilde{\mathcal{S}}, \tilde{Q}}(u, v)\right|$. To solve for $\alpha^{*}$ and $\beta^{*}$, we need to solve (15). To do so, we consider a breakdown to compute $M_{\mathcal{I}}$. Calculations are not difficult although a bit tedious. Details are given in Appendix B.

$$
\begin{aligned}
& M_{A}=0, \quad M_{H}=0 \\
& M_{B}=\min \left\{a_{0}-\alpha, \theta_{0}-\alpha+b_{1}-b_{0}, \theta_{1}-\alpha\right\}, \\
& M_{C}=\min \left\{b_{0}-\beta, \theta_{0}-\beta+a_{1}-a_{0}, \theta_{1}-\beta\right\}, \\
& M_{D}=\min \left\{b_{0}-\beta, \theta_{0}-\beta+a_{1}-a_{0}, \theta_{1}-\beta\right\}, \\
& M_{E}=\min \left\{a_{0}-\alpha, \theta_{0}-\alpha+b_{1}-b_{0}, \theta_{1}-\alpha\right\}, \\
& M_{F}=\min \left\{a_{0}-\alpha, \theta_{0}-\alpha+b_{1}-b_{0}, \theta_{1}-\alpha\right\}, \\
& M_{G}=\min \left\{b_{0}-\beta, \theta_{0}+\left(a_{1}-a_{0}\right)-\beta, \theta_{1}-\beta\right\} .
\end{aligned}
$$

Nothing can be concluded immediately in Section $\mathcal{S}$ in Figure $1\left(a_{0} \leqslant u \leqslant a_{1}\right.$ and $\left.b_{0} \leqslant v \leqslant b_{1}\right)$ since it cannot be distinguished which is larger: $f_{\alpha}(u, v)=\alpha+\left(u-a_{0}\right)$ or $f_{\beta}(u, v)=\beta+\left(v-b_{0}\right)$. However, consider two separate cases where a line $\alpha+\left(u-a_{0}\right)=\beta+\left(v-b_{0}\right)$ divides area $\mathcal{S}$ into two subareas:

$$
\mathcal{S}=\mathcal{S}_{1} \bigcup \mathcal{S}_{2}
$$

where $\mathcal{S}_{1}=\left\{f_{\alpha}(u, v) \leqslant f_{\beta}(u, v)\right\}$ and $\mathcal{S}_{2}=\left\{f_{\alpha}(u, v) \geqslant f_{\beta}(u, v)\right\}$.

$$
\begin{aligned}
& M_{\mathcal{S}_{1}}=\min \left(-\alpha+a_{0}, \theta_{0}-\alpha+b_{1}-b_{0}, \theta_{1}-\alpha\right) \\
& M_{\mathcal{S}_{2}}=\min \left(b_{0}-\beta, \theta_{0}-\beta+a_{1}-a_{0}, \theta_{1}-\beta\right)
\end{aligned}
$$

Define $\eta(\alpha)$ and $\phi(\beta)$ as follows

$$
\begin{aligned}
& \eta(\alpha)=\max \left(M_{B}, M_{E}, M_{F}, M_{\mathcal{S}_{2}}\right)=\min \left(-\alpha+a_{0}, \theta_{0}-\alpha+b_{1}-b_{0}, \theta_{1}-\alpha\right), \\
& \phi(\beta)=\max \left(M_{C}, M_{D}, M_{G}, M_{\mathcal{S}_{1}}\right)=\min \left(b_{0}-\beta, \theta_{0}-\beta+a_{1}-a_{0}, \theta_{1}-\beta\right) .
\end{aligned}
$$

Notice that (15) is decreasing in terms of $\alpha$ and $\beta$. In order to minimize the function, the constraints in (16) need to show that $\alpha+\beta=\theta_{0}+\theta_{1}$. However, it cannot be neglected that $\alpha+\beta \leqslant \min \left(a_{0}, b_{1}\right)+\min \left(a_{1}, b_{0}\right)$. So

$$
\alpha+\beta=\min \left\{\theta_{0}+\theta_{1}, \min \left(a_{0}, b_{1}\right)+\min \left(a_{1}, b_{0}\right)\right\}
$$

Let $\gamma=\min \left\{\theta_{0}+\theta_{1}, \min \left(a_{0}, b_{1}\right)+\min \left(a_{1}, b_{0}\right)\right\}$, which is known from the assumptions of the theorem; then $\beta$ can be rewritten as $\gamma-\alpha$ and $\phi(\beta)$ as

$$
\phi(\alpha)=\min \left(b_{0}+\alpha-\gamma, \theta_{0}+\alpha-\gamma+a_{1}-a_{0}, \theta_{1}+\alpha-\gamma\right)
$$

It remains to minimize over $\alpha$ and $\beta$

$$
\max _{(u, v) \in \mathcal{S}}\left\{\max \left(A^{\mathcal{S}, Q}-\min \left(f_{\alpha}(u, v), f_{\beta}(u, v)\right), 0\right)\right\}=\max (\eta(\alpha), \phi(\alpha))
$$

over $[0,1] \times[0,1]$ to find a value for $\alpha^{*}$. It can be seen that $\eta(\alpha)$ is decreasing in terms of $\alpha$, while $\phi(\alpha)$ is increasing in terms of $\alpha$. Moreover, if $\alpha^{*}$ is constrained to $\min _{\alpha}\{\max (\eta(\alpha), \phi(\alpha))\}, \alpha^{*}$ is obtained by setting $\eta(\alpha)=\phi(\alpha)$. Otherwise, the optimality would be violated since there would exist some $\alpha$ such that $\alpha>\alpha^{*}\left(\alpha<\alpha^{*}\right)$ where $\max (\eta(\alpha)$, $\phi(\alpha))=$ $\phi(\alpha)>\phi\left(\alpha^{*}\right)\left(\max (\eta(\alpha), \phi(\alpha))=\eta(\alpha)>\eta\left(\alpha^{*}\right)\right)$.

Therefore, $\alpha^{*}$ is the solution to the function $\eta(\alpha)=\phi(\alpha)$ and $\beta^{*}=\gamma-\alpha^{*}$. To conclude, $A^{\tilde{\mathcal{S}}}, \tilde{Q}$ is a copula in the set $\mathcal{C}$ and $C^{*}=A^{\widetilde{\mathcal{S}}}, \widetilde{Q}$ for the parameters $\alpha^{*}$ and $\beta^{*}$. 


\section{Example 5.}

Let $\mathcal{S}=\{(1 / 3,1 / 3),(5 / 6,5 / 6)\}$ and suppose $Q$ is the independence copula (i.e. $Q(u, v)=u v)$ then $Q(1 / 3,1 / 3)=1 / 9$ and $Q(5 / 6,5 / 6)=25 / 36$. Define $\widetilde{S}=\{(1 / 3,1 / 3),(5 / 6,5 / 6),(1 / 3,5 / 6),(5 / 6,1 / 3)\}$. From Theorem $2, \alpha^{*}$ is obtained by solving $\eta(\alpha)=\phi(\alpha)$ that is $\min (-\alpha+1 / 3,1 / 9-\alpha+1 / 2,25 / 36-\alpha)=\min (1 / 3-\beta, 1 / 9-\beta+1 / 2,5 / 6-\beta)$. We then obtain $1 / 3-\alpha=1 / 3-\beta$, equivalently $\alpha=\beta$. Recall that

$$
\beta=\min \{17 / 18, \min (1 / 3,5 / 6)+\min (5 / 6,1 / 3)\}-\alpha=2 / 3-\alpha
$$

Finally we obtain that

$$
\alpha^{*}=\beta^{*}=1 / 3 \text {. }
$$

Similar analysis can be done for the lower bound for a decreasing set.

This approach can be useful to approximate the lower or upper bound when they are not sharp (that is when they could not be attained by copulas). Although the work is done with two constraints a similar technique can be applied when there are more than two constraints on the copula. In this case, the derivations become very tedious and a numerical search may be more efficient.

Theorem 4 has direct applications in optimal portfolio selection. To be able to implement the optimal strategy, one needs a bound that is attainable by a copula (and not only a quasi copula). This was already used in Bernard, Boyle and Vanduffel [1], Bernard and Vanduffel [5] and in Bernard, Chen and Vandufel [2]. Let us give an example of application of Theorem 4 which extends the probability constraint considered in Boyle and Tian [6].

\section{Example 6.}

In Example 3 of Bernard, Boyle and Vanduffel [1], they consider a Black Scholes market and assume that an investor wants to achieve the same distribution $F$ as an investment in the risky asset $S_{T}$ but is subject to additional constraints ${ }^{6}$.

$$
\mathbb{P}\left(S_{T}<95, Y_{T}>100\right)=0.2 \text { and } \mathbb{P}\left(S_{T}<80, Y_{T}>100\right)=0.05
$$

It turns out that the optimal portfolio is obtained when the copula between $S_{T}$ and $Y_{T}$ is maximum. In their paper, they are able to solve explicitly for the optimum as the constraints on the copula amount to constraint in a compact set consisting of two points $\mathcal{S}:=\left\{\left(a_{0}, b_{0}\right):=\left(F_{S_{T}}(95), F_{Y_{T}}(100)\right),\left(a_{1}, b_{1}\right):=\left(F_{S_{T}}(80), F_{Y_{T}}(100)\right)\right\}$. This compact satisfies for example the conditions of Theorem 2 and thus the maximum bound on the set of constrained copulas on $\mathcal{S}$ is a copula and thus can lead to an explicit optimal strategy. In the case when the constraints (19) are on an increasing set, $a_{0}<a_{1}, b_{0}<b_{1}$ the exact optimal strategy cannot be constructed and Theorem 4 allows to construct the best approximate strategy.

\section{Acknowledgements}

C. Bernard acknowledges support from NSERC, from the Alexander von Humboldt Research Foundation and from the Society of Actuaries Centers of Actuarial Excellence Research Grant. Y. Liu and N. MacGillivray both acknowledge the research award associated with the President's Scholarship of Distinction from the University of Waterloo. J. Zhang was an exchange student from the Chinese University of Hong Kong at the time the research was conducted. We thank Steven Vanduffel for suggestions on an earlier draft as well as the editor Giovanni Puccetti and two anonymous referees.

6 We note that the feasibility of a particular constraint depends on the marginal distributions of $S_{T}$ and $Y_{T}$. This has been verified in our setting. 


\section{References}

[1] Bernard, C., Boyle, P.P., Vanduffel S. (2011). "Explicit Representation of Cost-Efficient Strategies”, Working paper available at SSRN.

[2] Bernard, C., Chen, J.S., Vanduffel S. (2013). “Optimal Portfolio under Worst-State Scenarios”, Quant. Finance, to appear.

[3] Bernard, C., Jiang, X., Vanduffel S. (2012). Note on“ Improved Fréchet bounds and model-free pricing of multi-asset options" by Tankov (2011)", J. of Appl. Probab., 49(3), 866-875.

[4] Bernard, C., Jiang, X., Wang R. (2013). "Risk Aggregation with Dependence Uncertainty", Working paper.

[5] Bernard, C., Vanduffel S. (2011). "Optimal Investment under Probability Constraints", AfMath proceedings.

[6] Boyle, P.P., and W. Tian. 2007, "Portfolio Management with Constraints," Math. Finance, 17(3), 319-343.

[7] Carley, H., Taylor, M.D. (2002). "A new proof of Sklar's Theorem” in C.M. Cuadras, J. Fortiana and J.A. RodriguezLallena, editors, Distributions with Given Marginals and Statistical Modelling, 29-34, Kluwer Acad. Publ., Dodrecht.

[8] Durante, F., Jaworski, P. (2010). "A new characterization of bivariate copulas" Comm. Statist. Theory Methods, 39(16), 2901-2912.

[9] Durante, F., Mesiar, R., Papini, P.-L., Sempi, C. (2007). "2-increasing binary aggregation operators", Inform. Sci., 177(1), 111-129.

[10] Embrechts, P., Puccetti, G. and Rüschendorf, L. (2013). "Model uncertainty and VaR aggregation". J. of Banking and Finance, 37(8), 2750-2764.

[11] Fréchet, M. (1951). "Sur les tableaux de corrélation dont les marges sont données,"Ann. Univ. Lyon Sect.A, Series $3,14,53-77$.

[12] Genest, C., Quesada-Molina, J.J., Rodriǵuez, J.A., Sempi, C. (1999). "A characterization of quasi-copulas", J. of Multivariate Anal., 69(2), 193-205.

[13] Grabisch, M., Marichal, J.-L., Mesiar, R., Pap, E. (2009). "Aggregation functions," Encyclopedia of Mathematics and its Applications. Cambridge University Press, New York, (No. 127).

[14] Hoeffding, W. (1940). "Masstabinvariante Korrelationstheorie," Schriften des mathematischen Instituts und des Instituts für angewandte Mathematik der Universität Berlin 5, 179-233.

[15] Kolesárová, A., Mordelová, J., Muel., E. (2004). "Kernel aggregation operators and their marginals," Fuzzy Sets Syst., 142(1), 35-50.

[16] Mai, J.-F., Scherer, J., (2012). "Simulating Copulas," World Scientific, Singapore.

[17] Meilijson, I., Nadas, A. (1979). "Convex majorization with an application to the length of critical paths," J. of Appl. Probab., 16, 671-677.

[18] Nelsen, R. (2006). "An introduction to Copulas”, 2nd edition, Springer series in Statistics.

[19] Nelsen, R., Quesada-Molina, J., Rodriguez-Lallena, J. and Úbeda-Flores, M. (2001). “Bounds on Bivariate Distribution Functions with Given Margins and Measures of Associations", Comm. Statist. Theory Methods. 30(6), 1155-1162.

[20] Nelsen, R., Quesada-Molina, J., Rodriguez Lallena, J. and Ubeda-Flores, M. (2004). “Best Possible Bounds on Sets of Bivariate Distribution Functions", J. of Multivariate Anal., 90, 348-358.

[21] Rachev, S.T. and Rüschendorf, L. (1994). "Solution of some transportation problems with relaxed or additional constraints", SIAM J. Control Optim., 32, 673-689.

[22] Rüschendorf, L. (1983). "Solution of a Statistical Optimization Problem by Rearrangement Methods", Biometrika, 30, 55-61.

[23] Sadooghi-Alvandi, S. M., Shishebor, Z., Mardani-Fard, H.A. (2013). "Sharp bounds on a class of copulas with known values at several points" Communications Statist. Theory Methods, 42(12), 2215-2228.

[24] Stoeber, J. and Czado, C. (2012). "Detecting regime switches in the dependence structure of high dimensional financial data", forthcoming in Comput. Statist. Data Anal..

[25] Tankov, P., (2011). "Improved Fréchet bounds and model-free pricing of multi-asset options", J. of Appl. Probab., 48, 389-403.

[26] Tchen, A. H., (1980). “Inequalities for distributions with given margins”, Ann. of Appl. Probab., 8, 814-827. 


\section{Appendix A: CALCULATIONS NEEDED FOR SIMPLIFYING $\pi_{A}$}

For $i \in \mathcal{I}$, the support of the copula on $\Gamma_{i}$ can be described by a set of points $\left(u, v_{i}(u)\right)$ where $v_{i}$ is going to be specified below. Aggregating the analysis together,

$$
\pi_{A}=P_{\mathcal{S}}+\sum_{i \in \mathcal{I}} P_{i}
$$

where $P_{\mathcal{S}}:=\int_{a_{0}}^{a_{1}} \int_{b_{0}}^{b_{1}} h\left(F_{X}^{-1}(u), F_{Y}^{-1}(v)\right) d Q(u, v)$, and $P_{i}:=\int_{\Gamma_{i}} h\left(u, v_{i}(u)\right) d u$. Precisely,

$$
\begin{aligned}
& P_{H}=\int_{0}^{Q\left(a_{0}, b_{0}\right)} h\left(F_{X}^{-1}(u), F_{Y}^{-1}(u)\right) d u \\
& P_{F}=\int_{Q\left(a_{0}, b_{0}\right)}^{Q\left(a_{0}, b_{1}\right)} h\left(F_{X}^{-1}(u), F_{Y}^{-1}\left(\phi_{F}^{-1}(u)\right)\right) d u \\
& P_{E}=\int_{Q\left(a_{0}, b_{1}\right)}^{a_{0}} h\left(F_{X}^{-1}(u), F_{Y}^{-1}\left(u+b_{1}-Q\left(a_{0}, b_{1}\right)\right)\right) d u \\
& P_{G}=b_{0} \int_{a_{0}}^{a_{1}} h\left(F_{X}^{-1}(u), F_{Y}^{-1}\left(Q\left(u, b_{0}\right)\right)\right) d u \\
& P_{B}=\left(1-b_{1}\right) \int_{a_{0}}^{a_{1}} h\left(F_{X}^{-1}(u), F_{Y}^{-1}\left(u-Q\left(u, b_{1}\right)+b_{1}\right)\right) d u \\
& P_{D}=\int_{a_{1}}^{a_{1}+b_{0}-Q\left(a_{1}, b_{0}\right)} h\left(F_{X}^{-1}(u), F_{Y}^{-1}\left(Q\left(a_{1}, b_{0}\right)+u-a_{1}\right)\right) d u \\
& P_{C}=\int_{a_{1}+b_{0}-Q\left(a_{1}, b_{0}\right)}^{a_{1}+b_{1}-Q\left(a_{1}, b_{1}\right)} h\left(F_{X}^{-1}(u), F_{Y}^{-1}\left(\phi_{C}^{-1}\left(u-a_{1}\right)\right)\right) d u \\
& P_{A}=\int_{a_{1}+b_{1}-Q\left(a_{1}, b_{1}\right)}^{1} h\left(F_{X}^{-1}(u), F_{Y}^{-1}(u)\right) d u
\end{aligned}
$$

where $\phi_{F}(v)=Q\left(a_{0}, v\right)$ and $\phi_{C}(v)=v-Q\left(a_{1}, v\right)$.

\section{Example of Bounds on a Bivariate Risk Measure}

Assume that $Q(u, v)=u v$ and that $h(u, v)=(\max (u, v)-K)^{+}$and let us compute the upper and lower bounds for $\pi(C)$. The support of the copula $A^{\mathcal{S}, Q}$ can be simplified. We find that

$$
\Gamma_{A}:=\left\{(u, v) \in A \mid u=v: u \geqslant a_{1}+b_{1}-a_{1} b_{1}, v \geqslant a_{1}+b_{1}-a_{1} b_{1}\right\}
$$

$\Gamma_{H}:=\left\{(u, v) \in H \quad \mid u=v: u \leqslant a_{0} b_{0}, v \leqslant a_{0} b_{0}\right\}, \Gamma_{G}:=\left\{(u, v) \in G \mid v=b_{0} u\right\}, \Gamma_{D}:=\left\{(u, v) \in D \mid v=u+a_{1}\left(1-b_{0}\right)\right\}$, $\Gamma_{Q}:=\left\{(u, v) \in Q \mid v=\frac{u-a_{1}}{1-a_{1}}\right\}, \Gamma_{F}:=\left\{(u, v) \in F \mid v=\frac{u}{a_{0}}\right\}, \Gamma_{E}:=\left\{(u, v) \in E \mid v=u+b_{1}\left(1-a_{0}\right)\right\}$, and $\Gamma_{B}:=\{(u, v) \in$ $\left.B \mid v=u\left(1-b_{1}\right)+b_{1}\right\}$. The expression for $\pi_{A}$ then writes as

$$
\begin{aligned}
\pi_{A}= & P_{\mathcal{S}}+\int_{0}^{a_{0} b_{0}} h\left(F_{X}^{-1}(u), F_{Y}^{-1}(u)\right) d u+\int_{a_{0} b_{0}}^{a_{0} b_{1}} h\left(F_{X}^{-1}(u), F_{Y}^{-1}\left(\frac{u}{a_{0}}\right)\right) d u \\
& +\int_{a_{0} b_{1}}^{a_{0}} h\left(F_{X}^{-1}(u), F_{Y}^{-1}\left(u+b_{1}\left(1-a_{0}\right)\right)\right) d u+b_{0} \int_{a_{0}}^{a_{1}} h\left(F_{X}^{-1}(u), F_{Y}^{-1}\left(b_{0} u\right)\right) d u \\
& +\left(1-b_{1}\right) \int_{a_{0}}^{a_{1}} h\left(F_{X}^{-1}(u), F_{Y}^{-1}\left(u\left(1-b_{1}\right)+b_{1}\right)\right) d u+\int_{a_{1}+b_{0}\left(1-a_{1}\right)}^{a_{1}+b_{1}\left(1-a_{1}\right)} h\left(F_{X}^{-1}(u), F_{Y}^{-1}\left(\frac{u-a_{1}}{1-a_{1}}\right)\right) d u \\
& +\int_{a_{1}}^{a_{1}+b_{0}\left(1-a_{1}\right)} h\left(F_{X}^{-1}(u), F_{Y}^{-1}\left(a_{1}\left(b_{0}-1\right)+u\right)\right) d u+\int_{a_{1}+b_{1}\left(1-a_{1}\right)}^{1} h\left(F_{X}^{-1}(u), F_{Y}^{-1}(u)\right) d u .
\end{aligned}
$$


On computing the double integral over the region $\mathcal{S}$, the expression can be rewritten strictly in terms of $d u$ and $d v$, since $Q$ is the independence copula.

$$
P_{\mathcal{S}}=\int_{a_{0}}^{a_{1}} \int_{b_{0}}^{b_{1}}\left(\max \left(\mu_{x}+\sigma_{x} \Phi^{-1}(u), \mu_{y}+\sigma_{y} \Phi^{-1}(v)\right)-K\right)^{+} d u d v
$$

\section{Appendix B: AUXILIARY CALCULATIONS TO PROVE THEOREM 4}

Section $\mathrm{A}$ in Figure 1: $u \geqslant a_{1}$ and $v \geqslant b_{1}$.

$$
\begin{aligned}
& f_{\alpha}(u, v)=\alpha+\left(u-a_{0}\right)+\left(v-b_{1}\right) \geqslant \theta_{1}+\left(u-a_{1}\right)+\left(v-b_{1}\right) \geqslant A^{\mathcal{S}, Q}(u, v) \\
& f_{\beta}(u, v)=\beta+\left(u-a_{1}\right)+\left(v-b_{0}\right) \geqslant \theta_{1}+\left(u-a_{1}\right)+\left(v-b_{1}\right) \geqslant A^{\mathcal{S}, Q}(u, v)
\end{aligned}
$$

where the first inequality in each expression follows from the Lipschitz property of $Q(u, v)$. Thus $A^{\mathcal{S}, Q}=A^{\tilde{\mathcal{S}}, \tilde{Q}}$ and $M_{A}=0$.

Section B in Figure 1: $a_{0} \leqslant u \leqslant a_{1}$ and $v \geqslant b_{1}$.

$$
\begin{aligned}
& f_{\alpha}(u, v)=\alpha+\left(u-a_{0}\right)+\left(v-b_{1}\right) \\
& f_{\beta}(u, v)=\beta+\left(v-b_{0}\right) \geqslant \theta_{1}+\left(v-b_{1}\right) \geqslant A^{\mathcal{S}, Q}(u, v)
\end{aligned}
$$

which implies that

$$
\begin{aligned}
M_{B} & =\max _{(u, v) \in B}\left\{\max \left(A^{S, Q}(u, v)-f_{\alpha}(u, v), 0\right)\right\} \\
& =\max _{(u, v) \in B}\left\{\left(\min \left\{\begin{array}{l}
u-f_{\alpha}(u, v), \theta_{0}+\left(u-a_{0}\right)+\left(v-b_{0}\right)-f_{\alpha}(u, v), \\
v-f_{\alpha}(u, v), \theta_{1}+\left(v-b_{1}\right)-f_{\alpha}(u, v),
\end{array}\right\}\right)^{+}\right\} \\
& =\max _{(u, v) \in B}\left\{\left(\min \left(-\alpha+a_{0}-v+b_{1}, \theta_{0}-\alpha+b_{1}-b_{0}, \theta_{1}-\alpha-u+a_{0}\right)\right)^{+}\right\} \\
& =\min \left\{-\alpha+a_{0}, \theta_{0}-\alpha+b_{1}-b_{0}, \theta_{1}-\alpha\right\}
\end{aligned}
$$

where in the third equality, $v-f_{\alpha}(u, v)$ is discarded because (17) implies $Q\left(a_{1}, b_{1}\right)=\theta_{1} \leqslant \min \left(a_{1}, b_{1}\right) \leqslant b_{1}$ so that $v \geqslant v+\theta_{1}-b_{1}=\theta_{1}+\left(v-b_{1}\right)$. Notice that the third equation is decreasing in terms of $u$ and $v$, so the maximum value can be obtained by the point $\left(a_{0}, b_{1}\right)$; this yields the fourth equality.

Section $C$ in Figure 1: $u \geqslant a_{1}$ and $b_{0} \leqslant v \leqslant b_{1}$.

$$
\begin{aligned}
& f_{\alpha}(u, v)=\alpha+\left(u-a_{0}\right) \geqslant \theta_{1}+\left(u-a_{1}\right) \geqslant A^{\mathcal{S}, Q}(u, v) \\
& f_{\beta}(u, v)=\beta+\left(u-a_{1}\right)+\left(v-b_{0}\right)
\end{aligned}
$$

which implies that $M_{C}=\max _{(u, v) \in C}\left\{\left(A^{\mathcal{S}, Q}(u, v)-f_{\beta}(u, v)\right)^{+}\right\}$

$$
\begin{aligned}
M_{C} & =\max _{(u, v) \in C}\left\{\left(\min \left\{\begin{array}{l}
u-f_{\beta}(u, v), \theta_{0}+\left(u-a_{0}\right)+\left(v-b_{0}\right)-f_{\beta}(u, v), \\
v-f_{\beta}(u, v), \theta_{1}+\left(u-a_{1}\right)-f_{\beta}(u, v)
\end{array}\right\}\right)^{+}\right\} \\
& =\max _{(u, v) \in C}\left\{\left(\min \left(-\beta+b_{0}-\left(u-a_{1}\right), \theta_{0}-\beta+a_{1}-a_{0}, \theta_{1}-\beta-\left(v-b_{0}\right)\right)\right)^{+}\right\} \\
& =\min \left\{-\beta+b_{0}, \theta_{0}-\beta+a_{1}-a_{0}, \theta_{1}-\beta\right\}
\end{aligned}
$$

where in the third equality, $u-f_{\beta}(u, v)$ is discarded because (17) shows that $u \geqslant u+\theta_{1}-a_{1}=\theta_{1}+\left(u-a_{1}\right)$. Notice that the third equation is decreasing in terms of $u$ and $v$, so the maximum value can be obtained by $\left(a_{1}, b_{0}\right)$. 
Similarly as in the Sections A, B and C considered above, we find the following results in the remaining sections $D$, E, $\mathrm{F}, \mathrm{G}$ and $\mathrm{H}$ from Figure 1.

Section $\mathcal{S}$ in Figure 1: $a_{0} \leqslant u \leqslant a_{1}$ and $b_{0} \leqslant v \leqslant b_{1}$.

$$
f_{\alpha}(u, v)=\alpha+\left(u-a_{0}\right), \quad f_{\beta}(u, v)=\beta+\left(v-b_{0}\right)
$$

$\mathcal{S}$ is divided into two subareas $\mathcal{S}=\mathcal{S}_{1} \cup \mathcal{S}_{2}$ where $\mathcal{S}_{1}=\left\{f_{\alpha}(u, v) \leqslant f_{\beta}(u, v)\right\}$ and $\mathcal{S}_{2}=\left\{f_{\alpha}(u, v) \geqslant f_{\beta}(u, v)\right\}$.

Subcase I: for $(u, v) \in \mathcal{S}_{1}, f_{\alpha}(u, v) \leqslant f_{\beta}(u, v)$,

$$
\begin{aligned}
M_{\mathcal{S}_{1}} & =\max _{(u, v) \in \mathcal{S}_{1}}\left\{\left(A^{\mathcal{S}, Q}-f_{\alpha}(u, v)\right)^{+}\right\} \\
& =\max _{(u, v) \in \mathcal{S}_{1}}\left\{\left(\min \left\{\begin{array}{l}
u-f_{\alpha}(u, v), v-f_{\alpha}(u, v), \theta_{1}-f_{\alpha}(u, v), \\
\theta_{0}+\left(u-a_{0}\right)+\left(v-b_{0}\right)-f_{\alpha}(u, v)
\end{array}\right\}\right)^{+}\right\} \\
& =\max _{(u, v) \in \mathcal{S}_{1}}\left\{\left(\min \left\{\begin{array}{l}
-\alpha+a_{0}, v-\alpha-\left(u-a_{0}\right), \theta_{0}-\alpha+v-b_{0}, \\
\theta_{1}-\alpha-\left(u-a_{0}\right)
\end{array}\right\}\right)\right\} \\
& =\min \left(-\alpha+a_{0}, b_{1}-\alpha, \theta_{0}-\alpha+b_{1}-b_{0}, \theta_{1}-\alpha\right) \\
& =\min \left(-\alpha+a_{0}, \theta_{0}-\alpha+b_{1}-b_{0}, \theta_{1}-\alpha\right)
\end{aligned}
$$

where the penultimate equality holds since the third equation is decreasing in terms of $u$ and increasing in terms of $v$ and so the maximum value can be obtained by point the $\left(a_{0}, b_{1}\right)$. For the last equality, $b_{1}-\alpha$ is discarded because (17) shows that $b_{1}-\alpha \geqslant b_{1}-\alpha+\theta_{0}-b_{0}$.

Subcase II: for $(u, v) \in \mathcal{S}_{2}, f_{\alpha}(u, v) \geqslant f_{\beta}(u, v)$, we can prove similarly as in $\mathcal{S}_{1}$ that $M_{\mathcal{S}_{2}}=\min \left(b_{0}-\beta, \theta_{0}-\beta+a_{1}-\right.$ $\left.a_{0}, \theta_{1}-\beta\right)$ 